Pacific Journal of Mathematics

SYMMETRIC PERPENDICULARITY IN HILBERT

PAuL JOSEPH KelLy AND Lowell J. Paid 


\title{
SYMMETRIC PERPENDICULARITY IN HILBERT GEOMETRIES
}

\author{
P. J. KeLly and L. J. PAIge
}

1. Introduction. A hilbert plane geometry [2] can be generated in the following way. Let $K$ be a simple, closed, convex curve in the euclidean plane and $H$ its open interior. If $a$ and $b$ are any two points in $H$, they determine a line $a \times b^{1}$ which intersects $K$ in a pair of points $u$ and $v$. With $R$ denoting cross-ratio, the hilbert distance from $a$ to $b$ is defined by

$$
h(a, b)=k|\log R(a, b ; u, v)|,
$$

where $k$ is an arbitrary positive constant. The region $H$ is then a metric set with respect to $K$. Under the additional requirement that $K$ contain at most one segment, $H$ defines a hilbert plane geometry in which any pair of points are uniquely connected by a geodesic, and these geodesics are open straight lines. If $K$ is an ellipse, then the hilbert geometry coincides with the well-known Klein model of hyperbolic geometry.

Perpendicularity in $H$ is defined through the idea of distance. If $p$ and $\xi$ are any point and line respectively, then a point $f$ on $\xi$ is a "foot of $p$ on $\xi$ " if $h(p, f) \leq h(p, x)$ for all points $x$ on $\xi$. A line $\eta$, intersecting $\xi$, is perpendicular to $\xi$ if every point on $\eta$ has the point of intersection, $\xi \times \eta$, as a foot on $\xi$. Under this definition, there is no need for the perpendicularity of $\eta$ to $\xi$ to imply the perpendicularity of $\xi$ to $\eta$. The aim here is to show that when perpendicularity is always symmetric, the hilbert geometry is hyperbolic.

As before, let $p$ and $\xi$ be any point and line in $H$, and let $\eta$ be a line passing through $p$ and intersecting $K$ in the points $u$ and $v$. It can be shown quite simply that a necessary and sufficient condition for $\eta$ to be perpendicular to $\xi$ is that a pair of supporting lines exist, one at $u$ and one at $v$, intersecting at a point $w$ on $\xi$ [1]. If $\eta$ is perpendicular to $\xi$, then the previous statement implies that $\eta$ is also perpendicular to every line through $w$ which is a secant to $K$. When such a secant cuts $K$ at points $m$ and $n$, then symmetry of perpendicularity requires that a supporting line exist at $m$, and one at $n$, such that the two intersect on $\eta$.

\footnotetext{
${ }^{1}$ Here and henceforth the line joining $a$ and $b$ will be indicated by $a \times b$, and symmetrically the point of intersection on lines $\xi$ and $\eta$ by $\xi \times \eta$.

Received February 1, 1952.
}

Pacific J. Math. 2 (1952), 319-322 
2. The family $\boldsymbol{F}$. The foregoing facts suggested the independent problem of identifying the following family of curves.

Family $F$ : Every curve $C$ in $F$ is a simple, closed, convex curve. If supporting lines at $p$ and $q$ on $C$ meet at $w$, and $\xi$ is any secant through $w$, cutting $C$ at $m$ and $n$, then supporting lines at $m$ and $n$ exist, intersecting on $p \times q$.

We are going to show that the family $F$ consists of all triangles and ellipses.

LеммA 1. If a curve $C$ in $F$ contains a straight line segment then the curve is a triangle.

Proof. Let $a$ and $b$ denote the end points of a segment contained in $C$, and take $p$ to be a regular point (a point of $C$ with unique supporting line) of $C$ which is not on $\xi=a \times b$. If $\sigma$ denotes the supporting line at $p$, assume that $q=\sigma \times \xi$ is not a point of $C$. Because the secants to $C$ through $q$ form a continuum, while the corner points (points possessing more than one supporting line) of $C$ are denumerable, there exists a secant $\eta$, through $q$, such that its intersections with $C$ are two regular points $m$ and $n$. But by the definition of $C$, the unique supporting lines at $m$ and $n$ must intersect on $p \times b$ and also on $p \times a$. Hence they intersect at $p$, which contradicts the fact that $p$ is regular. Therefore the intersection $\sigma \times \xi$ is a point of $C$, and so is either $a$ or $b$. Suppose it to be $a$. The segment from $a$ to $p$ is then part of $C$. Let $c$ denote the other end of the largest segment contained in $C$ and containing the segment from $a$ to $p$. Let $r$ be a regular point of $C$, not on $\xi$ nor on $\gamma=a \times p$, and let $\delta$ be the supporting line at $r$. By the same reasoning as before, the points $\xi \times \delta$ and $\delta \times \gamma$ must lie on $C$, and hence are the points $b$ and $c$ respectively. Thus $C$ is the triangle $a, b, c$.

LEмма 2. If a curve $C$ in $F$ contains a corner point, then the curve is a triangle.

Proof. Let $p$ be a corner point on $C$, with $\delta_{1}$ and $\delta_{2}$ two supporting lines at p. Assume: $(*)$ that no supporting line contains two points of $C$. Let $q$ and $r$ be any two regular points of $C$, with $\sigma$ and $\eta$ denoting their respective supporting lines. Set $u_{i}=\sigma \times \delta_{i}(i=1,2)$ and $v=\eta \times(p \times q)$. Because of $(*)$, the line $u_{i} \times r$ is a secant, and intersects $C$ again at a point $s_{i}(i=1,2)$. By the definition of $C$, at $s_{i}$ a supporting line exists which intersects $\eta$ at a point of $p \times q$, namely at the point $v$. But because of $(*)$, the point $v$ is exterior to $C$. In addition to $\eta$, then, there can be only one other supporting line through $v$. Hence the lines $v \times s_{1}$ and $v \times s_{2}$ are the same, which contradicts (*). Because $(*)$ is false, $C$ contains a segment, and so, by Lemma 1 , it is a triangle. 
THEOREM l. The family $F$ consists of all ellipses and all nondegenerate triangles.

Proof. If $C$ contains a segment or a corner point then it is a triangle; so suppose $C$ to be strictly convex and differentiable. Let $p_{1}$ and $p_{2}$ be two points of $C$ such that the supporting lines, $\sigma_{1}$ and $\sigma_{2}$, at $p_{1}$ and $p_{2}$ are parallel. Introduce a rectangular reference frame so that $p_{1}$ is the origin, $\sigma_{1}$ is the y-axis, and with $p_{2}$ lying in the first quadrant. Take $\theta$ to denote the acute angle between the the line $\eta=p_{1} \times p_{2}$ and the $\mathrm{x}$-axis, and let $\sigma_{2}$ be the line $x=k$. A vertical chord of $C$, lying on the line $\sigma(x)$ through $(x, 0)$, is cut by $\eta$ into an upper and lower segment such that the ratio of their lengths, $\mu(x)$, is constant for all $x$ on the interval $\langle 0, k\rangle$. To prove this, let $T$ be the affinity $y^{\prime}=-x \tan \theta+y$, $x^{\prime}=x$, taking $C$ into a new convex curve $C^{\prime}$. Under $T$, the line $\eta$ goes into the $x$-axis, which seperates $C^{\prime}$ into an upper curve $y_{1}=f_{1}(x)$ and a lower curve $y_{2}=f_{2}(x)$. Because $T$ preserves distance on any vertical line, the ratio $\mu(x)$ equals $f_{1}(x) / f_{2}(x)$. The line $\sigma(x)$ is a secant to $C$ through $\sigma_{1} \times \sigma_{2}$; hence the tangents to $C$, at its intersections with $\sigma(x)$, are lines which intersect on $\bar{\eta}$. Then $C^{\prime}$ has the property that the tangents at $\left(x, f_{1}(x)\right)$ and $\left(x, f_{2}(x)\right)$ intersect on the $x$-axis. From simple triangle relations it follows that

$$
\frac{f_{1}^{\prime}(x)}{f_{1}(x)}=\frac{f_{2}^{\prime}(x)}{f_{2}(x)}
$$

for $x$ on $\langle 0, k\rangle$. If $a$ is fixed, and $x$ is variable, on $\langle 0, k\rangle$, then the equality

$$
\int_{a}^{x} \frac{f_{1}^{\prime}(x) d x}{f_{1}(x)}=\int_{a}^{x} \frac{f_{2}^{\prime}(x) d x}{f_{2}(x)}
$$

shows that

$$
\frac{f_{1}(x)}{f_{2}(x)}=\frac{f_{1}(a)}{f_{2}(a)}
$$

and hence that $\mu(x)$ is a constant. The original curve $C$, therefore, has the property that if a line joins the contact points of two tangents which are parallel in a direction $\alpha$, then the line cuts all chords parallel to $\alpha$ in a ratio which is constant $($ with $\alpha$ ). But then it is known that the ratio is unity for all directions and that the curve is an ellipse [3]. Since it is easily shown that either a triangle or an ellipse does belong to the family $F$, the theorem is complete. In particular, it may be noted that the property of family $F$, applied to strictly convex curves, characterizes the ellipse. 
3. Symmetric perpendicularity. The answer to the original problem is now clear. When perpendicularity is symmetric in a hilbert geometry, then the curve $C$ belongs to the family $F$. Since $C$ can have at most one segment, it is not a triangle, and hence is is an ellipse. Therefore the geometry is hyperbolic. Thus we have proved the following theorem.

THEOREM 2. The hilbert geometries in which perpendicularity is symmetric are the hyperbolic geometries.

The result obviously extends to higher dimensions. Perpendicularity refers to lines in the same plane. When the perpendicularity is symmetric, every plane section of the gauge surface $K$ is an ellipse; hence $K$ is an ellipsoid which defines a higher dimensional hyperbolic geometry.

\section{REFERENCES}

1. H. Buseman and P. Kelly, Projective geometry and projective metrics (In Press), Chapter 3, Section 7. 1930.

2. David Hilbert, Grundlagen der Geometrie, Anhang I, Seventh Edition, Leipzig,

3. Tadahiko Kubota, On a characteristic property of the ellipse, Tohoku Math. J. 9 (1916), 148-151.

University of California, Santa Barbara

University of California, Los Angeles 


\title{
PACIFIC JOURNAL OF MATHEMATICS
}

\section{EDITORS}

\section{R. M. RobINSON \\ University of California \\ Berkeley 4, California}

\author{
*R. P. Dilwor TH \\ California Institute of Technology \\ Pasadena 4, California
}

\author{
E. F. BECKENBACH, Managing Editor \\ University of California \\ Los Angeles 24, California
}

${ }^{*}$ During the absence of Herbert Busemann in 1952.

\section{ASSOCIATE EDITORS}

$\begin{array}{llll}\text { R. P. DILWORTH } & \text { P. R. HALMOS } & \text { B } \emptyset \text { RGE JESSEN } & \text { J. J. STOKER } \\ \text { HERBERT FEDERER } & \text { HEINZ HOPF } & \text { PAUL LÉVY } & \text { E. G. STRAUS } \\ \text { MARSHALL HALL } & \text { R. D. JAMES } & \text { GEORGE PÓLYA } & \text { KÖSAKU YOSIDA }\end{array}$

\section{SPONSORS}

UNIVERSITY OF BRITISH COLUMBIA

CALIFORNIA INSTITUTE OF TECHNOLOGY

U NIVERSITY OF CALIFORNIA, BERKELEY

UNIVERSITY OF CALIFORNIA, DAVIS

UNIVERSITY OF CALIFORNIA, LOS ANGELES

UNIVERSITY OF CALIFORNIA, SANTA BARBARA

OREGON STATE COLLEGE

UNIVERSITY OF OREGON

\author{
UNIVERSITY OF SOUTHERN CALIFORNIA \\ STANFORD UNIVERSITY \\ WASHINGTON STATE COLLEGE \\ UNIVERSITY OF WASHINGTON \\ AMERICAN MATHEMATICAL SOCIETY \\ NATIONAL BUREAU OF STANDARDS, \\ INSTITUTE FOR NUMERICAL ANALYSIS
}

Mathematical papers intended for publication in the Pacific Journal of Miathematics should be typewritten (double spaced), and the author should keep a complete copy. Manuscripts may be sent to any of the editors. All other communications to the editors should be addressed to the managing editor, E. F. Beckenbach, at the address given above.

Authors are entitled to receive 100 free reprints of their published papers and may obtain additional copies at cost.

The Pacific Journal of Mathematics is published quarterly, in March, June, September, and December, by the University of California, Berkeley 4, California. The price per volume (4 numbers) is $\$ 8.00$; single issues, $\$ 2.50$. Special price to individual faculty members of supporting institutions and to individual members of the American Mathematical Society: $\$ 4.00$ per volume; single issues, $\$ 1.25$.

Subscriptions, orders for back numbers, and changes of address should be sent to the publishers, University of California Press, Berkeley 4, California.

Printed at Ann Arbor, Michigan. Entered as second class matter at the Post Office, Berkeley, California.

\section{UNIVERSITY OF CALIFORNIA PRESS • BERKELEY AND LOS ANGELES}




\section{Pacific Journal of Mathematics}

\section{Vol. 2, No. $3 \quad$ March, 1952}

Lars V. Ahlfors, Remarks on the Neumann-Poincaré integral equation .... 271

Leonard P. Burton, Oscillation theorems for the solutions of linear, nonhomogeneous, second-order differential systems ............ 281

Paul Civin, Multiplicative closure and the Walsh functions . . . . . . . . . . . 291

James Michael Gardner Fell and Alfred Tarski, On algebras whose factor algebras are Boolean .................................. 297

Paul Joseph Kelly and Lowell J. Paige, Symmetric perpendicularity in Hilbert geometries .................................. 319

G. Kurepa, On a characteristic property of finite sets .............. 323

Joseph Lehner, A diophantine property of the Fuchsian groups ......... 327

Donald Alan Norton, Groups of orthogonal row-latin squares ........... 335

R. S. Phillips, On the generation of semigroups of linear operators ....... 343

G. Piranian, Uniformly accessible Jordan curves through large sets of relative harmonic measure zero ........................ 371

C. T. Rajagopal, Note on some Tauberian theorems of $O . S z \tilde{A} ; s z \ldots \ldots \ldots 377$

Halsey Lawrence Royden, Jr., A modification of the Neumann-Poincaré method for multiply connected regions .................... 385

George H. Seifert, A third order irregular boundary value problem and the associated series ...................................... 395

Herbert E. Vaughan, Well-ordered subsets and maximal members of ordered sets....

Hans F. Weinberger, An optimum problem in the Weinstein method for eigenvalues.

Shigeki Yano, Note on Fourier analysis. XXXI. Cesàro summability of Fourier series. 